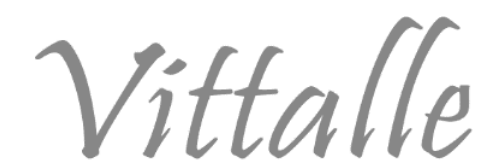

\title{
Correlação entre desfechos na saúde cardiorrespiratória e exposição a poluentes atmosféricos - um estudo na megacidade de São Paulo
}

\author{
Rodrigo Mascarenhas Pires de Godoy, Karina Camasmie Abe*, Simone Georges El \\ Khouri Miraglia
}

Laboratório de Economia, Saúde e Poluição Ambiental, Instituto de Ciências Ambientais, Químicas e Farmacêuticas, Universidade Federal de São Paulo - UNIFESP, Diadema, SP, Brasil

\section{Histórico do Artigo \\ Recebido em: \\ $10 / 08 / 2021$ \\ Aceito em: \\ $02 / 11 / 2021$}

\section{Palavras-chave:}

Material particulado; São

Paulo; impactos na saúde; poluição do ar; saúde cardiorrespiratória

Keywords:

Particulate matter; São Paulo; health impacts; air pollution; cardiorespiratory health

\begin{abstract}
RESUMO
O objetivo deste trabalho foi analisar os efeitos na saúde cardiorrespiratória da população do município de São Paulo pela exposição aos poluentes atmosféricos material particulado $\left(\mathrm{MP}_{10}\right), \mathrm{NO}_{2}, \mathrm{SO}_{2}$ e $\mathrm{O}_{3}$ durante os anos de 2010 a 2018. Para tal, foram levantados dados de mortalidade e morbidade por doenças respiratórias (CID-10, J00 - J99) e por doenças cardiovasculares (CID-10, I00 - I99), disponíveis nos bancos de dados do DATASUS e dados diários das concentrações de $\mathrm{MP}_{10}, \mathrm{NO}_{2}, \mathrm{SO}_{2}$ e $\mathrm{O}_{3}$ obtidos da plataforma Qualar da CETESB Por meio de análises descritivas preliminares pôde-se constatar o perfil sazonal das ocorrências de morbimortalidade por doenças cardiorrespiratórias, onde verifica-se os picos de casos nos meses de outono e inverno. Por fim, analisou-se estatisticamente os parâmetros de estudo por testes de Correlação de Pearson medindo o grau de interdependência entre duas varáveis. $\mathrm{O} \mathrm{NO}_{2}$ foi o único poluente atmosférico que apresentou uma correlação positiva significativa com todos os efeitos deletérios em saúde, enquanto o $\mathrm{MP}_{10}$ apresentou forte interdependência com os desfechos em saúde, exceto quando relacionado com os casos de internações cardiovasculares. Entre os parâmetros climáticos analisados destaca-se a correlação entre a temperatura mínima e os parâmetros de saúde, em especial os óbitos cardiovasculares. Esse estudo colaborou para destacar a importância da atualização de políticas públicas relacionadas à qualidade do ar e mobilidade urbana, colocando em destaque os efeitos deletérios à saúde cardiorrespiratória, pela exposição aos poluentes atmosféricos e as vantagens da melhoria da qualidade do ar, em grandes centros urbanos.
\end{abstract}

Correlation between cardiorespiratory health outcomes and exposure to air pollutants - a study in the megacity of São Paulo

\section{ABSTRACT}

The objective of this study was to analyze the effects on cardiorespiratory health of the population of the city of São Paulo by the exposure to the atmospheric pollutants particulate matter $\left(\mathrm{PM}_{10}\right), \mathrm{NO}_{2}, \mathrm{SO}_{2}$ and $\mathrm{O}_{3}$ during the years 2010 to 2018 . Mortality and morbidity data due to respiratory diseases were collected (ICD-10, J00 - J99) and for cardiovascular diseases (ICD-10, I00 - I99), available in the DATASUS databases and daily data on $\mathrm{PM}_{10}, \mathrm{NO}_{2}, \mathrm{SO}_{2}$ and $\mathrm{O}_{3}$ concentrations, obtained from the Qualar platform of CETESB. Through preliminary descriptive analyses, it was possible to verify the seasonal profile of the occurrences of morbidity and mortality due to cardiorespiratory diseases, where the peaks of cases in the autumn and winter months were observed. Finally, the studied parameters were statistically analyzed using Pearson's correlation tests, measuring the degree of interdependence between two variables. $\mathrm{NO}_{2}$ was the only air pollutant that showed a significant positive correlation with all harmful effects on health, whereas $\mathrm{PM}_{10}$ showed a strong interdependence with health outcomes, except when related to cases of cardiovascular hospitalizations. Among the climate parameters analyzed, the correlation between minimum temperature and health parameters, especially cardiovascular deaths, stands out. This study collaborated to high spot the importance of updating public policies related to air quality and urban mobility, high lighting the harmful effects on cardiorespiratory health due to exposure to air pollutants and the advantages of improving air quality in large urban centers.

\footnotetext{
* Autor correspondente: karina.camasmie @ gmail.com (Abe K.C.)
} 


\section{Introdução}

Uma grande parcela dos seres vivos necessita de ar para sua sobrevivência, incluindo os seres humanos. Através da respiração, obtemos e aproveitamos o oxigênio presente no ar atmosférico, no entanto, além dos gases necessários à manutenção da vida, outras partículas e gases nocivos aos seres humanos são retidos no nosso corpo durante o processo e possuem efeitos à saúde $(1,2)$.

Com o rápido processo de industrialização e a disseminação de veículos automotores nas últimas décadas, houve um incremento na emissão antrópica de poluentes atmosféricos e, consequentemente, uma degradação da qualidade do ar, principalmente nos grandes centros urbanos. Por essa razão, a comunidade científica intensificou seus esforços em estudar os efeitos da poluição atmosférica na saúde $(1,2)$. Dentre muitos desses poluentes, destacam-se o monóxido de carbono $(\mathrm{CO})$, o dióxido de enxofre $\left(\mathrm{SO}_{2}\right)$, o dióxido de nitrogênio $\left(\mathrm{NO}_{2}\right)$, ozônio troposférico $\left(\mathrm{O}_{3}\right)$ e o material particulado (MP), como principais focos dos estudos da área $(2,3)$.

Historicamente, os poluentes atmosféricos são associados, principalmente a complicações respiratórias e cardiovasculares, como constatado em diversos estudos (310), no entanto, esses componentes também estão relacionados a doenças neurológicas (11-14) e por complicações no processo de gestação (15-18), entre outros efeitos deletérios. A poluição do ar afeta todas as regiões, grupos socioeconômicos e faixas etárias (2).

O município de São Paulo é um dos maiores centros urbanos do mundo, totalizando uma população estimada de 12.396 .372 habitantes em 2021 (19). Devido à dimensão de sua frota automotiva (9,1 milhões de veículos), esses são responsáveis por $97 \%$ da emissão de $\mathrm{CO}, 74 \%$ de hidrocarbonetos, $62 \%$ de óxidos de nitrogênio e $40 \%$ de MP, poluentes aos quais a população está exposta gerando diversos impactos na saúde pública em termos de doenças respiratórias e cardiovasculares, concentrações associadas a perdas de cerca de 231.692 anos de vida, anualmente $(3,7,25)$.

A partir dos resultados desses estudos evidenciou-se a importância de ferramentas de análises do impacto à saúde da exposição aos poluentes atmosféricos em grandes centros urbanos. A identificação e quantificação dos efeitos deletérios dos poluentes atmosféricos na saúde pública consistem em importante fator norteador de políticas públicas que protejam a população mais vulnerável exposta aos contaminantes atmosféricos. Assim, objetivou-se com esse estudo analisar a associação entre parâmetros de morbimortalidade por doenças cardiorrespiratórias da população do município de São Paulo (MSP) pela exposição aos poluentes atmosféricos durante o período de 2010 a 2018.

\section{Material e métodos}

\section{Área de Estudo}

A cidade de São Paulo está localizada a $23^{\circ} 32^{\prime} 50^{\prime \prime} \mathrm{S}$ e $46^{\circ} 38^{\prime} 09^{\prime \prime} \mathrm{O}$, no estado de São Paulo, Brasil. A área total do município é de $1.521,1 \mathrm{~km}^{2}$ (2018) e está a uma altitude de 799m acima do nível do mar, com um clima subtropical úmido. Sua população total é de aproximadamente 11.811.516 habitantes (2019) e o Índice de Desenvolvimento Humano Regional é de 0,805 (2010), com 678 unidades de saúde pública. Sua frota veicular é de aproximadamente 8.295.645 automóveis (2018), a maior do Brasil (19, 20).

\section{Dados de Saúde}


Os dados de morbimortalidade foram levantados do banco de dados da plataforma online do Departamento de Informática do Sistema Único de Saúde (DATASUS) e tratados, incialmente, pelo Software Tabwin com posterior processo de filtragem feito nos softwares estatísticos IBM SPSS ${ }^{\circledR}$ versão 21 e Microsoft Excel®, versão 2016.

Disponibilizados em lotes anuais, os dados de mortalidade foram levantados a partir do Sistema de informações de Mortalidade (SIM) e selecionados para o período de janeiro de 2010 a dezembro de 2017 e para o MSP (código 3550308 - IBGE). Os óbitos então foram restringidos pela classificação do CID-10. As doenças foram triadas por causa principal do óbito sendo causa respiratória (CID-10, série J) e causa cardiovascular (CID10 , série I) as causas de mortalidade selecionadas para o presente estudo.

Os dados de morbidade foram obtidos através do Sistema de Informações Hospitalares (SIH) do DATASUS e são disponibilizados em lotes mensais, devido ao grande número de ocorrências. Foram levantados os dados relativos ao período de janeiro de 2010 a dezembro de 2018. Os métodos de filtragem e restrição dos casos foram análogos aos descritos dos dados de mortalidade.

A população total do município de São Paulo foi obtida a partir de dados demográficos da plataforma online da Prefeitura de São Paulo, esse dado foi posteriormente utilizado para calcular as taxas de cada desfecho em saúde estudado (caso /100 mil hab.) (22).

\section{Dados dos Poluentes}

Os dados dos poluentes atmosféricos foram obtidos a partir da plataforma de Qualidade do Ar da CETESB (Qualar). As médias diárias dos poluentes $\mathrm{MP}_{10}, \mathrm{O}_{3}, \mathrm{NO}_{2}$ e $\mathrm{SO}_{2}$ foram levantadas da rede automática de monitoramento para o período de janeiro de 2010 a dezembro de 2018.

As estações da rede automática são capazes de realizar medições horárias da concentração atmosférica dos poluentes. Para se ter uma média diária válida, cada estação deve atender ao requisito de registrar ao menos 16 medições horárias (2/3 da medição diária).

A partir dessa informação, inicialmente, filtrou-se as estações de monitoramento relevantes para o estudo pela sua geolocalização, excluindo as estações situadas fora do município de São Paulo (MSP); o outro critério de seleção das estações era apresentar ao menos $90 \%$ dos dados válidos. Ao fim desse processo, dez estações de monitoramento foram selecionadas, ao todo. Essas estações foram divididas em conjuntos referentes ao poluente analisado.

- $\mathrm{MP}_{10}$ : Estações Cerqueira César, Congonhas, Grajau-Parelheiros, Nossa Senhora do Ó e Parque Dom Pedro II;

- $\mathrm{O}_{3}$ : Estações Cidade Universitária - USP - Ipen, Ibirapuera, Mooca, Nossa Senhora do Ó, Parque Dom Pedro II, Pinheiros e Santana;

- $\mathrm{NO}_{2}$ : Estações Cerqueira César, Cidade Universitária - USP - Ipen, Congonhas, Ibirapuera, Parque Dom Pedro II e Pinheiros;

- $\mathrm{SO}_{2}$ : Cerqueira César e Congonhas.

Por fim, extraímos as médias simples das medições diárias das estações, obtendo um valor médio diário para a concentração de cada poluente no MSP.

Os valores para as médias anuais são obtidos a partir dos dados diários de cada poluente e apresentados conforme padrão média das concentrações diárias (MAA - anual) \pm desvio padrão (DP) conforme apresentado na tabela 3. 


\section{Dados Meteorológicos}

Os dados meteorológicos foram levantados do Sistema de Monitoramento Agrometeorológico (Agritempo). A busca foi limitada ao estado de São Paulo onde foi selecionada a Estação Meteorológica: São Paulo - São Paulo (IAG), o período selecionado foi entre 01/01/2010 à 31/12/2018. Os parâmetros utilizados no estudo foram Temperatura Mínima $\left({ }^{\circ} \mathrm{C}\right)$, Temperatura Média $\left({ }^{\circ} \mathrm{C}\right)$ e Precipitação $(\mathrm{mm})$.

\section{Análise de Correlação}

Os dados de saúde foram pré-tratados em Software Tabwin com posterior filtragem nos softwares estatísticos IBM SPSS $®$ versão 21 e Microsoft Excel®, versão 2016.

Inicialmente, considerando a hipótese de igualdade estatística entre os dados do início e final do período levantado, foram realizados testes $t$-Student para verificar diferenças estatísticas nos dados de desfecho em saúde (morbimortalidade cardiorrespiratória) e nos dados de poluentes atmosféricos entre os anos de 2010 e 2017/18.

Em um segundo momento, estimou-se o grau de associação existente entre a flutuação diária dos casos de morbimortalidade cardiorrespiratória, as variações diárias na concentração dos poluentes atmosféricos e os parâmetros climáticos. Para isso empregouse a análise por Correlação de Pearson definindo um nível de significância $(\mathrm{p} \leq 0,05)$ e assumindo a normalidade na distribuição dos dados amostrais, com base no Teorema do Limite Central (23). Com esses parâmetros ajustados, as amostras foram analisadas em pares, o número diário de internações e óbitos por doenças cardiovasculares e respiratórias foram as variáveis dependentes e as médias diárias dos parâmetros climáticos e dos poluentes atmosféricos foram as variáveis independentes. $\mathrm{O}$ valor do coeficiente de correlação de Pearson ( $\mathrm{r}_{\text {pearson }}$ ) indica o grau de relação linear entre as duas variáveis e são classificados de acordo com os intervalos de valores convencionados por Cohen (32), valores absolutos de $r_{\text {pearson }}$ entre 0,1 - 0,3 são classificados como correlação fraca, entre $0,3-0,5$ são considerados moderados e valor absolutos maiores que 0,5 são considerados como uma interdependência forte das variáveis.

Os testes estatísticos foram realizados em software IBM SPSS® versão 26, com a aplicação dos modelos nativos do programa.

\section{Resultados e Discussão}

\section{Dados de Saúde - Análise Descritiva}

No período analisado, percebeu-se os valores mais elevados das médias mensais durantes os meses de outono e inverno para todos os desfechos em saúde, de acordo com a Tabela 1.

O valor mais elevado da média mensal de internações por doenças cardiovasculares foi de 190,70 internações, correspondente aos meses de agosto, já para os casos de morbidade por doenças respiratórias, a maior média mensal foi de 206,93 internações correlato aos meses de abril. A maior média mensal de óbitos por doenças cardiovasculares foi durante os meses de junho com valor da média sendo de 73,8. Por fim, em relação aos óbitos por doença respiratória a maior média mensal foi durante os meses de julho com valor igual a 32,61 (Tabela 1).

Esses dados refletem e confirmam as tendências já verificadas em outros artigos internacionais e nacionais, em que os meses mais frios e com menor precipitação, estão associados com maior número de desfechos deletérios à saúde cardiorrespiratória e concentração de poluentes (24-26). 
Tabela 1 - Internações e óbitos por doenças cardiorrespiratórias (média \pm desvio padrão (DP)) por meses do ano. Período analisado: de 2010 a 2018 para dados de internações e de 2010 a 2017 para dados de óbitos, município de São Paulo. Destaque em negrito para o maior valor

\begin{tabular}{ccccc}
\hline MÊS & $\begin{array}{c}\text { Internações por } \\
\text { Doença } \\
\text { Cardiovascular } \\
\text { (média } \pm \mathbf{D P} \text { ) }\end{array}$ & $\begin{array}{c}\text { Internações por } \\
\text { Doença } \\
\text { Respiratória } \\
\text { (média } \pm \mathbf{D P})\end{array}$ & $\begin{array}{c}\text { Óbitos por Doença } \\
\text { Cardiovascular } \\
\text { (média } \pm \mathbf{D P} \text { ) }\end{array}$ & $\begin{array}{c}\text { Óbitos por Doença } \\
\text { Respiratória } \\
\text { (média } \pm \mathbf{D P} \text { ) }\end{array}$ \\
\hline Janeiro & $169,38 \pm 65,21$ & $101,78 \pm 23,43$ & $58,76 \pm 8,58$ & $23,17 \pm 5,58$ \\
Fevereiro & $168,55 \pm 46,81$ & $126,39 \pm 29,83$ & $58,89 \pm 9,64$ & $24,20 \pm 5,86$ \\
Março & $173,81 \pm 46,68$ & $179,48 \pm 38,82$ & $58,00 \pm 8,62$ & $23,76 \pm 6,76$ \\
Abril & $172,59 \pm 59,40$ & $\mathbf{2 0 6 , 9 3} \pm \mathbf{4 8 , 0 0}$ & $62,71 \pm 9,08$ & $26,61 \pm 7,09$ \\
Maio & $179,54 \pm 47,46$ & $179,27 \pm 38,00$ & $68,27 \pm 9,47$ & $29,56 \pm 7,17$ \\
Junho & $181,88 \pm 52,21$ & $166,33 \pm 33,32$ & $\mathbf{7 3 , 8 3} \pm \mathbf{1 0 , 2 4}$ & $31,69 \pm 6,94$ \\
Julho & $183,49 \pm 49,13$ & $147,63 \pm 32,38$ & $72,32 \pm 10,38$ & $\mathbf{3 2 , 6 1} \pm \mathbf{6 , 8 2}$ \\
Agosto & $\mathbf{1 9 0 , 7 0} \pm \mathbf{5 4 , 7 3}$ & $152,01 \pm 30,87$ & $67,70 \pm 9,79$ & $29,53 \pm 5,86$ \\
Setembro & $184,72 \pm 52,06$ & $146,20 \pm 30,06$ & $65,56 \pm 9,45$ & $27,83 \pm 5,92$ \\
Outubro & $182,12 \pm 52,91$ & $143,40 \pm 31,81$ & $62,83 \pm 8,84$ & $25,92 \pm 5,71$ \\
Novembro & $170,33 \pm 49,63$ & $135,67 \pm 28,52$ & $60,40 \pm 9,31$ & $24,83 \pm 5,90$ \\
Dezembro & $152,82 \pm 55,96$ & $111,82 \pm 37,21$ & $61,19 \pm 9,26$ & $25,24 \pm 5,54$ \\
\hline
\end{tabular}

Fonte: elaborada pelos autores com base nos dados do DATASUS.

Analisando a Tabela 2, nota-se um valor máximo para os casos de óbitos no ano de 2016 e, em 2017, para os casos de internações respiratórias, curiosamente as médias de internações cardiovasculares tiveram seu pico em 2010, no primeiro ano estudado, apontando uma possível melhora no quadro desse desfecho na saúde. Ademais, pode-se especular um possível aumento no número de internações respiratórias e óbitos com o decorrer dos anos, no entanto, análises estatísticas adicionais futuras precisão ser realizadas para a corroboração dessa hipótese.

Tabela 2 - Internações e óbitos por doenças cardiorrespiratórias (média \pm desvio padrão (DP)) por ano. Período analisado: de 2010 a 2018 para dados de internações e de 2010 a 2017 para dados de óbitos, município de São Paulo. Destaque em negrito para o maior valor

\begin{tabular}{ccccc}
\hline ANO & $\begin{array}{c}\text { Internações por } \\
\text { Doença } \\
\text { Cardiovascular } \\
\text { (média } \pm \text { DP) }\end{array}$ & $\begin{array}{c}\text { Internações por } \\
\text { Doença } \\
\text { Respiratória } \\
\text { (média } \pm \mathbf{D P} \text { ) }\end{array}$ & $\begin{array}{c}\text { Óbitos por Doença } \\
\text { Cardiovascular } \\
\text { (média } \pm \mathbf{D P} \text { ) }\end{array}$ & $\begin{array}{c}\text { Óbitos por Doença } \\
\text { Respiratória } \\
\text { (média } \pm \mathbf{D P} \text { ) }\end{array}$ \\
\hline 2010 & $\mathbf{1 5 7 , 1 6} \pm \mathbf{3 8 , 0 6}$ & $176,70 \pm 48,04$ & $62,30 \pm 9,97$ & $23,81 \pm 5,92$ \\
2011 & $156,53 \pm 40,31$ & $178,47 \pm 64,76$ & $63,23 \pm 10,68$ & $25,48 \pm 5,55$ \\
2012 & $147,58 \pm 43,76$ & $185,72 \pm 347,37$ & $60,77 \pm 10,30$ & $25,14 \pm 6,48$ \\
2013 & $153,81 \pm 45,74$ & $172,79 \pm 62,77$ & $64,34 \pm 10,41$ & $26,39 \pm 6,80$ \\
2014 & $144,78 \pm 43,53$ & $165,58 \pm 46,22$ & $64,04 \pm 10,19$ & $27,91 \pm 6,57$ \\
2015 & $143,26 \pm 42,89$ & $166,79 \pm 43,91$ & $64,69 \pm 10,34$ & $28,33 \pm 7,26$ \\
2016 & $154,66 \pm 51,92$ & $187,99 \pm 59,40$ & $\mathbf{6 8 , 5 2} \pm \mathbf{1 1 , 0 2}$ & $\mathbf{3 0 , 1 9} \pm \mathbf{7 , 5 7}$ \\
2017 & $148,53 \pm 40,75$ & $\mathbf{1 9 2 , 1 0} \pm \mathbf{5 7 , 6 1}$ & $65,93 \pm 10,81 * * *$ & $29,49 \pm 6,86 * * *$ \\
2018 & $141,59 \pm 48,92$ & $176,75 \pm 61,77$ & - & - \\
\hline
\end{tabular}

Fonte: elaborada pelos autores com base nos dados do DATASUS. $* * * p<0,001$. 


\section{Dados dos Poluentes Atmosféricos}

A localização geográfica das estações de monitoramento é de extrema importância para uma melhor compreensão e análise dos dados obtidos. Pode-se notar, pela Figura 1, uma concentração das estações na região central do MSP e a presença de estações distribuídas nas zonas periféricas do município.
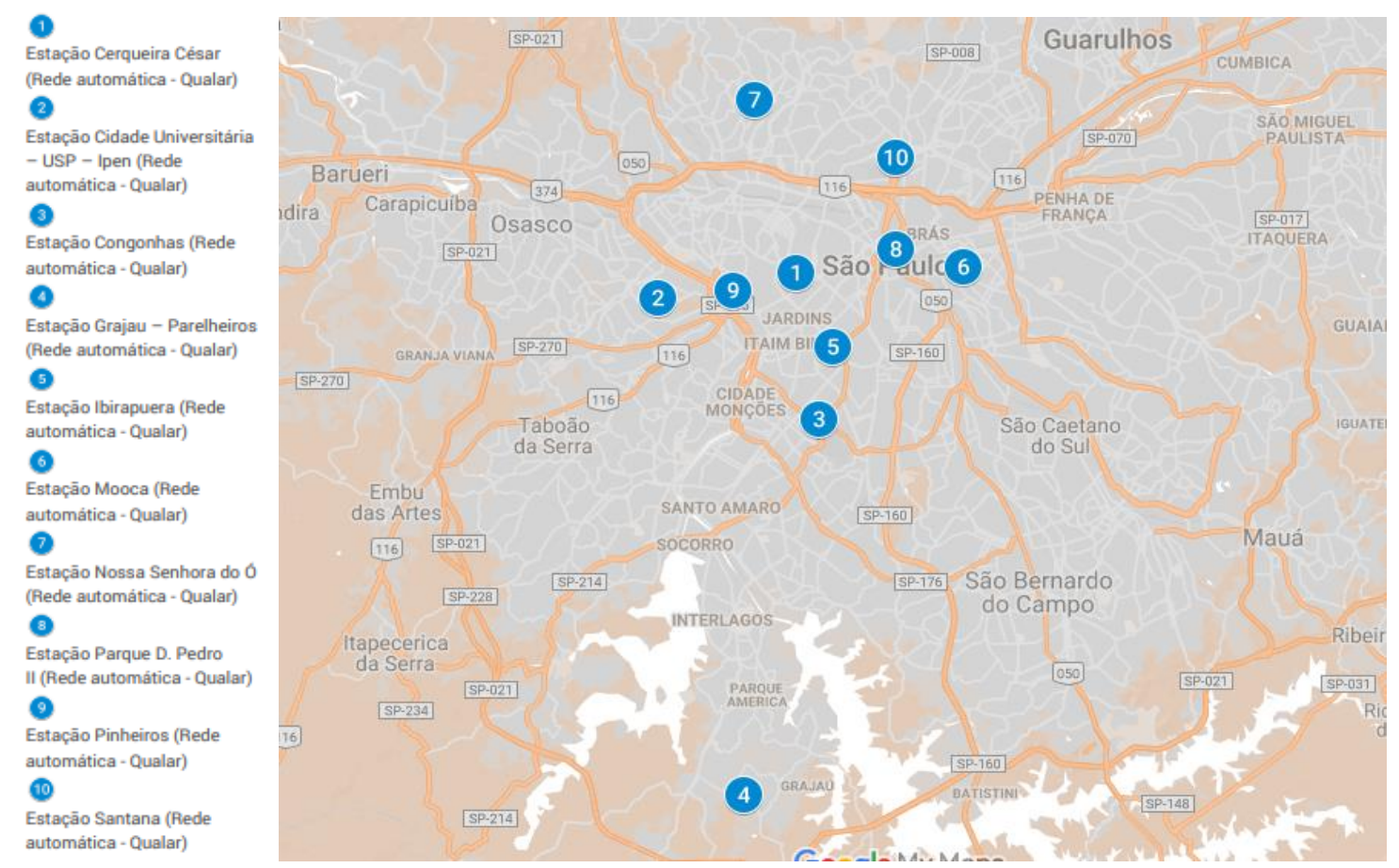

Figura 1 - Mapa das estações de monitoramento de poluentes utilizados no levantamento do estudo. Fonte: Elaborado pelos autores com base no Google Maps.

Verificou-se que ao início do período de estudo, em 2010, a concentração média diária de $\mathrm{MP}_{10}$ foi de $35,75 \pm 18,14 \mu \mathrm{g} / \mathrm{m}^{3}$, e ao final do período esse valor foi de $30,04 \pm 15,69$ $\mu \mathrm{g} / \mathrm{m}^{3}$, representando uma redução de aproximadamente $16,0 \%$. Os valores máximos diários também seguiram essa tendência de redução, foram de $114,00 \mu \mathrm{g} / \mathrm{m}^{3}$, em 2010 , para $85,80 \mu \mathrm{g} / \mathrm{m}^{3}$ em 2018. Pela Figura 2, pode-se observar as séries temporais dos poluentes estudados, e pela linha de tendência ajustada da série, obteve-se uma suavização das variações diárias, ou seja, reduzindo o ruído gerado pelos outliers. Também pela mesma figura pode-se ver uma suave tendência de redução na concentração de $\mathrm{NO}_{2}$ atmosférico e uma redução mais acentuada para o parâmetro $\mathrm{SO}_{2}$.

De acordo com os Padrões Estaduais de Qualidade do Ar, estabelecidos pelo Decreto Estadual $n^{\circ} 59113$ de 23/04/2013 (26), nota-se pela Tabela 3 que o MSP está próximo de se adequar à classificação MI3 nos padrões do $\mathrm{MP}_{10}$ atmosférico (MAA $\leq 30 \mu \mathrm{g} / \mathrm{m}^{3}$ ), visto que para os anos de 2015 a 2018, obtivemos valores muito próximos ou até mesmo inferiores ao padrão determinado. Em relação aos outros poluentes, o $\mathrm{SO}_{2}$ apresentou valores adequados ao padrão final $(\mathrm{PF})$ para ambos valores diários e média anual (MAA $\leq 20 \mu \mathrm{g} / \mathrm{m}^{3}$ ), já o $\mathrm{NO}_{2}$ apresentou um quadro de não atendimento aos padrões estabelecidos, não acatando aos valores de MAA, enquanto o $\mathrm{O}_{3}$ não pôde ser avaliado pela falta de parâmetros diários ou anuais. 
Tabela 3 - Análise descritiva dos poluentes atmosféricos para o município de São Paulo (média das concentrações diárias \pm desvio padrão) por ano e valores dos parâmetros MI3 e PF dos Padrões Estaduais de Qualidade do Ar. Período analisado: 2010 a 2018

\begin{tabular}{|c|c|c|c|c|}
\hline ANO & $\begin{array}{c}\text { Concentração de } \\
\mathrm{MP}_{10}(\mathrm{MAA} \pm \mathrm{DP}) \\
\left(\mu \mathrm{g} / \mathrm{m}^{\mathbf{3}}\right)\end{array}$ & $\begin{array}{c}\text { Concentraçãa de } \\
\mathrm{O}_{3}(\mathrm{MAA} \pm \mathrm{DP}) \\
\left(\mu \mathrm{g} / \mathrm{m}^{3}\right)\end{array}$ & $\begin{array}{c}\text { Concentração de } \\
\mathrm{NO}_{2}(\mathrm{MAA} \pm \mathrm{DP}) \\
\left(\mu \mathrm{g} / \mathrm{m}^{3}\right)\end{array}$ & $\begin{array}{c}\text { Concentração de } \\
\mathrm{SO}_{2}(\mathrm{MAA} \pm \mathrm{DP}) \\
\left(\mu \mathrm{g} / \mathrm{m}^{3}\right)\end{array}$ \\
\hline 2010 & $35,75 \pm 18,14$ & $62,73 \pm 28,80$ & $82,08 \pm 35,71$ & $6,52 \pm 2,96$ \\
\hline 2011 & $36,73 \pm 16,15$ & $66,01 \pm 30,43$ & $76,09 \pm 31,45$ & $6,25 \pm 2,39$ \\
\hline 2012 & $35,64 \pm 14,51$ & $70,30 \pm 31,41$ & $75,49 \pm 28,80$ & $4,52 \pm 2,43$ \\
\hline 2013 & $34,06 \pm 15,25$ & $56,62 \pm 27,44$ & $64,82 \pm 25,17$ & $3,97 \pm 1,89$ \\
\hline 2014 & $34,12 \pm 15,75$ & $69,41 \pm 34,20$ & $72,37 \pm 28,87$ & $3,94 \pm 1,97$ \\
\hline 2015 & $30,06 \pm 12,75$ & $66,13 \pm 32,29$ & $67,42 \pm 26,48$ & $2,93 \pm 1,73$ \\
\hline 2016 & $28,94 \pm 13,08$ & $62,73 \pm 29,39$ & $63,41 \pm 24,90$ & $2,41 \pm 1,66$ \\
\hline 2017 & $29,10 \pm 14,20$ & $61,61 \pm 29,05$ & $70,03 \pm 26,11$ & $2,58 \pm 1,26$ \\
\hline 2018 & $30,04 * * * \pm 15,69$ & $62,65 \pm 24,80$ & $69,58 * * * \pm 28,00$ & $2,14 * * * \pm 1,25$ \\
\hline $\begin{array}{c}\text { Padrão } \\
\text { MI3 }\end{array}$ & 30 (MAA) & $120(8 \mathrm{~h})$ & 45 (MAA) & 20 (MAA) \\
\hline Padrão PF & 20 (MAA) & $100(8 \mathrm{~h})$ & 40 (MAA) & - \\
\hline
\end{tabular}

*** $\mathrm{p}<0,001$.

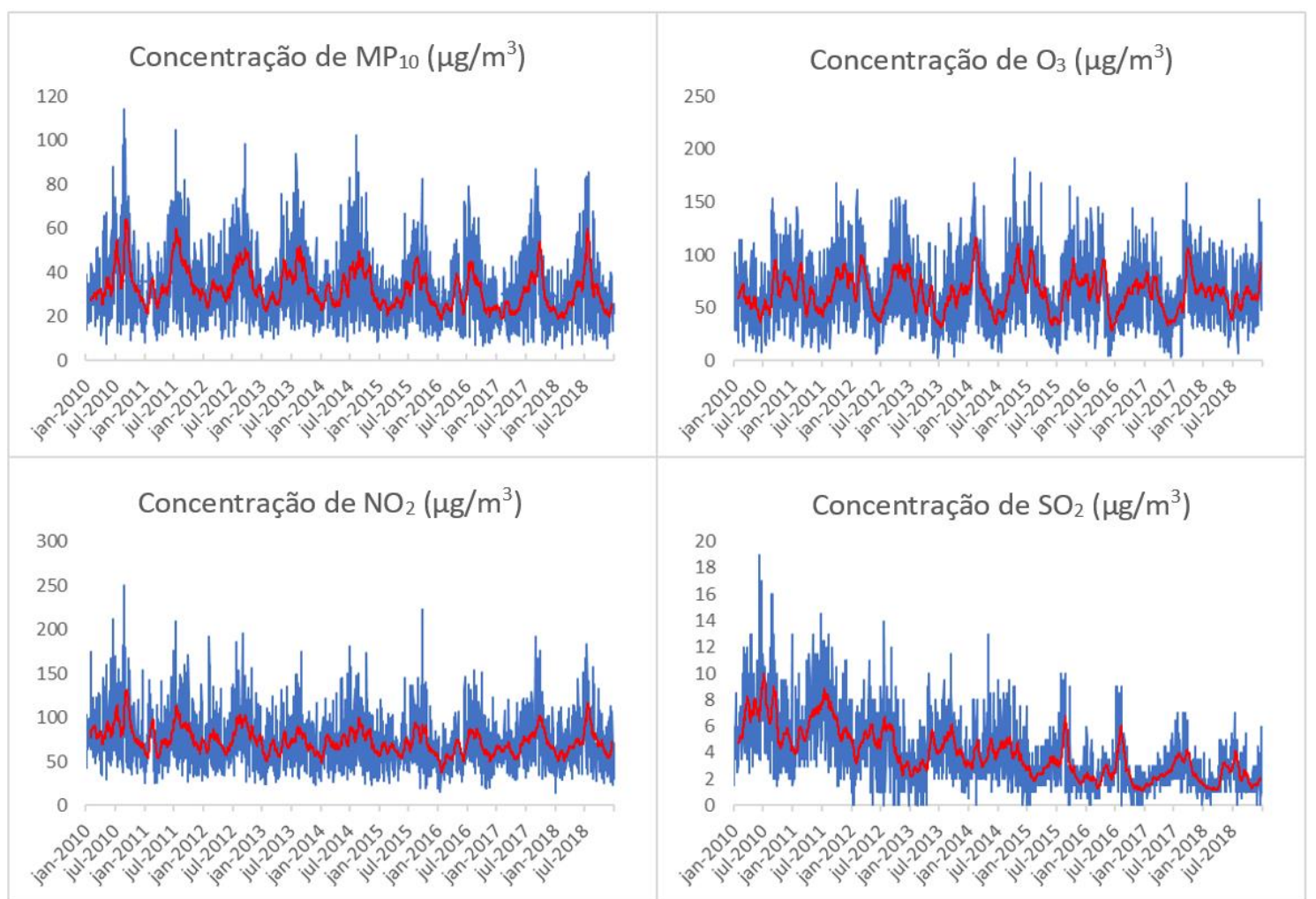

Figura 2 - Séries temporais das concentrações diárias dos poluentes analisados durante o período de 2010 a 2019 para o município de São Paulo (médias diárias). Linha de tendência (vermelha) ajustada por média móvel com período de 30 dias.

Medidas limitadoras de emissão de poluentes atmosféricos e melhorias dos combustíveis, tanto por avanços tecnológicos quanto por iniciativas e políticas públicas, como o Programa de Controle da Poluição do Ar por Veículos Automotores 
(PROCONVE) foram efetivas para o controle da emissão de poluentes por veículos automotivos. O PROCONVE está vigente desde 1986 e possui diversas fases relacionadas, tendo como alvo os veículos automotores leves e pesados (28). Ao longo do período analisado (2010 - 2018) duas fases do programa foram totalmente implantadas (L-5 e L-6), enquanto a fase L-7, está em fase de adaptação para que seus requisitos sejam atendidos até $2022(28,29)$. Em complemento ao PROCONVE, em 2002, entrou em vigor o Programa de Controle da Poluição do Ar por Motociclos e Veículos Similares (PROMOT), tal programa visa controlar as emissões da categoria de motociclos e similares, veículos amplamente utilizados no segmento de prestação de serviços em áreas urbanas. O PROMOT também é segmentado, em fases denominadas de "M". Ao todo, desde o início do programa, 4 fases foram aprovadas e implementadas (M-1 a M-4) e uma quinta fase foi aprovada em 2019, com previsão de implantação inicial para 2023 (30, 31). Essas ações auxiliaram no controle da emissão de poluentes atmosféricos pela queima de combustíveis utilizados em veículos automotores leves, pesados e motociclos. Desse modo, apesar do crescimento da frota veicular no MSP, como visto na Figura 3, essas medidas foram um importante contrapeso no balanço da emissão dos poluentes, em especial do $\mathrm{MP}_{10}$ atmosférico, propiciando um quadro com tendência de redução da concentração atmosférica de $\mathrm{MP}_{10}$ no MSP (figura 2). Na tabela 4 é possível verificar que a população do MSP cresceu cerca de 3,6\% entre 2010 a 2018 enquanto a frota veicular cresceu mais de $24 \%$, para o mesmo período. Notadamente, os níveis dos poluentes atmosféricos $\mathrm{MP}_{10}, \mathrm{SO}_{2}$ e $\mathrm{NO}_{2}$, sofreram um decréscimo nesse período, com exceção do $\mathrm{O}_{3}$, poluente de origem secundária, que necessita de radiação ultravioleta para ser formado. Pode-se notar, também, uma branda redução na taxa de motorização da população ao longo do período analisado, sendo um importante fator de estudo devido a sua relação com diversos fatores, incluindo a poluição do ar. A elevada taxa revela uma deficiência em termos de políticas públicas que favoreçam o transporte público em detrimento ao individual.

Ainda há muito o que ser melhorado, mas é possível detectar que políticas públicas, de longo prazo, nessa área, possuem resultados atrelados à melhoria dos índices de poluentes urbanos.

Tabela 4 - Valores absolutos de população e frota veicular e taxa de motorização para o município de São Paulo (MSP), entre os anos de 2010 a 2018

\begin{tabular}{cccc}
\hline \multirow{2}{*}{ ANO } & \multirow{2}{*}{ POPULAÇÃO } & $\begin{array}{c}\text { FROTA } \\
\text { VEICULAR }\end{array}$ & $\begin{array}{c}\text { TAXA DE MOTORIZAÇ̃̃O DA } \\
\text { POPULAÇÃO (HAB/VEİCULOS) }\end{array}$ \\
\hline 2010 & 11.253 .503 & 4.617 .635 & 2,437 \\
2011 & 11.303 .626 & 4.739 .236 & 2,385 \\
2012 & 11.353 .750 & 4.839 .921 & 2,346 \\
2013 & 11.403 .873 & 4.971 .813 & 2,294 \\
2014 & 11.453 .996 & 5.160 .727 & 2,219 \\
2015 & 11.504 .120 & 5.318 .961 & 2,163 \\
2016 & 11.554 .243 & 5.442 .775 & 2,123 \\
2017 & 11.604 .366 & 5.582 .546 & 2,079 \\
2018 & 11.654 .490 & 5.729 .252 & 2,034 \\
\hline
\end{tabular}

Fonte: PREFEITURA DE SÃO PAULO, 2018 (20); DENATRAN, 2018 (21).

\section{Análise de Correlação}

Conforme a Tabela 5, os dados de óbitos cardiovasculares apresentam um grau de interdependência fraco com os poluentes $\mathrm{MP}_{10}\left(\mathrm{r}_{\text {pearson }}=0,224\right), \mathrm{NO}_{2}\left(\mathrm{r}_{\text {pearson }}=0,180\right) \mathrm{e}$ os parâmetros meteorológicos precipitação $\left(r_{\text {pearson }}=-0,103\right)$ e temperatura média $\left(r_{\text {pearson }}\right.$ 
$=-0,256)$, sendo que a temperatura mínima foi o único parâmetro analisado que apresentou uma interdependência negativa e de grau moderado $\left(r_{\text {pearson }}=-0,318\right)$. Um perfil muito semelhante pode ser notado nas correlações para os óbitos respiratórios, onde o $\mathrm{MP}_{10}$ e o $\mathrm{NO}_{2}$ apresentaram uma pequena interdependência linear com esse desfecho, representado pelos coeficientes 0,218 e 0,141, respectivamente. Ainda nesse desfecho, percebe-se uma dependência linear fraca e negativa com os parâmetros de temperatura.

Em relação aos dados de morbidade cardiovascular, apenas o $\mathrm{NO}_{2}$ apresentou um grau de correlação significativo com esse desfecho $\left(r_{\text {pearson }}=0,116\right)$, todos os outros parâmetros podem ser considerados estatisticamente insignificantes quando correlacionados a esse desfecho em saúde $\left(\left|\mathbf{r}_{\text {pearson }}\right|<0,1\right)$.

Os testes de Correlação para internações por causas respiratórias apresentaram um grau de interdependência linear fraco com todos os parâmetros independentes analisados. No caso dos pares com as variáveis meteorológicas, as correlações foram de caráter negativo, assim como a análise conjunta com o $\mathrm{O}_{3}$.

Tabela 5 - Análise do grau de Correlação entre desfechos em saúde e o parâmetros atmosféricos/meteorológicos. Período de análise: Óbitos (2010 - 2017); Internações $(2010-2018)$

\begin{tabular}{|c|c|c|c|c|c|c|c|}
\hline \multirow[b]{2}{*}{$\begin{array}{l}\text { Desfechos em } \\
\text { Saúde }\end{array}$} & \multicolumn{7}{|c|}{ Correlações de Pearson } \\
\hline & $\mathbf{M P}_{10}$ & $\mathbf{O}_{3}$ & $\mathrm{NO}_{2}$ & $\mathrm{SO}_{2}$ & $\begin{array}{c}\text { TEMPERATURA } \\
\text { MÍNIMA }\end{array}$ & $\begin{array}{c}\text { TEMPERATURA } \\
\text { MÉDIA }\end{array}$ & PRECIPITAÇÃO \\
\hline $\begin{array}{c}\text { Óbitos } \\
\text { Cardiovasculares }\end{array}$ & $0,224 * *$ & $-0,086 * *$ & $0,180^{* *}$ & $0,079 * *$ & $-0,318 * *$ & $-0,256^{* *} *$ & $-0,103 * *$ \\
\hline $\begin{array}{c}\text { Óbitos } \\
\text { Respiratórios }\end{array}$ & $0,218 * *$ & $-0,051 * *$ & $0,141^{* *}$ & $-0,012$ & $-0,192 * *$ & $-0,154 * *$ & $-0,089 * *$ \\
\hline $\begin{array}{c}\text { Internações } \\
\text { Cardiovasculares }\end{array}$ & $0,078^{* *}$ & $-0,029$ & $0,116^{* *}$ & $0,057 * *$ & $-0,070 * *$ & $-0,037 *$ & $-0,012$ \\
\hline $\begin{array}{l}\text { Internações } \\
\text { Respiratórias }\end{array}$ & $0,235^{* *}$ & $-0,168 * *$ & $0,239 * *$ & $0,248 * *$ & $-0,122 * *$ & $-0,115^{* *}$ & $-0,111 * *$ \\
\hline
\end{tabular}

Em paralelo a diversos estudos já publicados pela comunidade científica, vemos que os resultados obtidos no estudo corroboram outros estudos da literatura internacional e nacional. Assim como observado no estudo de Costa et al. (33), também na capital paulista, constatou-se associações positivas entre $\mathrm{MP}_{10}, \mathrm{NO}_{2}$ e $\mathrm{CO}$ e a mortalidade respiratória de idosos no MSP. Já Ribeiro et al. (34), identificaram uma relação entre casos de internação hospitalar por câncer do aparelho respiratório e o fato de residir em regiões com grande tráfego veicular. Ademais, outros estudos corroboram a relação entre o $\mathrm{MP}_{10}$ e os casos de óbitos por doenças cardiorrespiratórias, como visto por Abe et al. (25), Pinheiro et al. (35) e Nascimento et al. (36). Já estudando os efeitos do $\mathrm{NO}_{2}$ na saúde, Ribeiro et al. (37) identificaram uma associação entre esse poluente e internações por doenças do trato respiratório.

Situações semelhantes também são encontradas em outras metrópoles, por exemplo, em Hong Kong, na China, verificou-se um aumento nos casos de óbitos cardiorrespiratórios relacionados ao aumento das concentrações de $\mathrm{MP}_{2,5}$ e $\mathrm{MP}_{10}$ (38). Nesse mesmo país, Zheng et al. (39), também observaram associação positiva entre casos de morbidade respiratória e os poluentes $\mathrm{MP}_{10}, \mathrm{SO}_{2}$ e $\mathrm{NO}_{2}$. Na Alemanha, um estudo de corte verificou uma forte associação entre poluentes do ar e desfechos como doenças respiratórias e mortalidade por câncer de pulmão (40). Semelhantemente, uma revisão americana 
verificou que a exposição de longo prazo ao $\mathrm{NO}_{2}$, está associada a um maior risco de mortalidade cardiovascular e respiratória (41).

Em relação ao $\mathrm{O}_{3}$, alguns estudos mostram fraca associação com desfechos na mortalidade por todas as causas (42) e concluem que a maioria das evidências associaram o $\mathrm{O}_{3}$ para efeitos de curto prazo, especialmente nos sistemas respiratório e cardiovascular. As evidências relacionadas aos efeitos de longo prazo são menos conclusivas (43). No presente estudo, foram detectadas correlações entre o $\mathrm{O}_{3}$ e os desfechos em saúde com óbitos cardiovasculares e respiratórios e internações respiratórias de forma fraca e negativa, porém, nesse estudo foi realizada a análise de longo prazo desse poluente.

Não foram constatadas associações de $\mathrm{SO}_{2}$ com os desfechos de saúde analisados. Isso muito provavelmente se deve ao fato de as associações deste poluente com indicadores de morbi-mortalidade serem encontradas com níveis de concentração elevados de $\mathrm{SO}_{2}$, o que não foi constatado em São Paulo, onde a média de concentração no período analisado variou de 6,52 a $2,14 \mu \mathrm{g} / \mathrm{m}^{3}$. Para se ter uma referência comparativa, no estudo de $\mathrm{Wu}$ et. al. (44) a média de $\mathrm{SO}_{2}$ foi de $122,08 \mu \mathrm{g} / \mathrm{m}^{3}$ causando 884 óbitos e 27.854 casos (44).

Conhecendo-se os efeitos deletérios causados pelos poluentes atmosféricos, uma possível medida que auxiliaria na melhora da qualidade do ar no MSP seria o incentivo à compra de carros elétricos, por exemplo, investindo em pontos públicos de recarga (eletropostos) e no desenvolvimento de tecnologias nacionais para baratear os custos desses veículos. O primeiro semestre de 2020 representou um aumento de $221 \%$ nas vendas de carros elétricos no Brasil, em comparação com o mesmo período de 2019 (45), e a Associação Brasileira do Veículo Elétrico (ABVE) prevê um aumento de 300 a 500\% nas vendas dos próximos 5 anos, no entanto isso representa apenas uma parcela irrisória do mercado de automóveis (cerca de $1 \%$ ).

Estudos que correlacionam os efeitos na saúde e os custos econômicos gerados pelos poluentes atmosféricos são menos frequentes apesar de fornecerem um subsídio eficiente para políticas públicas (44-47). No Brasil, sobre os veículos importados dessa categoria cobram-se 35\% de Imposto de Importação, 25\% de IPI, 12\% de ICMS e 9,25\% de PIS/ Cofins, tornando esses veículos inacessíveis à grande parcela da população. A Medida Provisória $\mathrm{n}^{\circ} 843$ (Projeto Rota 2030), de 2018 é uma tentativa de incentivar o desenvolvimento de novas tecnologias e inovações voltadas a eficiência energética, no entanto, o foco principal do Projeto não é o incentivo para o uso do carro elétrico ou híbrido, se mostrando obsoleto do ponto de vista mundial (48-50). Outra iniciativa privada foi tomada por montadoras de veículos, pela instalação do primeiro corredor de eletropostos ligando São Paulo, Rio de Janeiro, Vitória, Florianópolis e Curitiba. No entanto Zaneti (51), afirma que o sucesso dos carros elétricos em países desenvolvidos está diretamente relacionado aos investimentos públicos realizados na mobilidade e energia. Os automóveis elétricos e híbridos comprovadamente podem reduzir a emissão de poluentes atmosféricos (50-53), além disso alguns estudos já esboçam planos de instalação de eletropostos em munícipios (55-56), porém, incentivos fiscais também refletem uma grande importância na popularização dessa categoria de veículo. Além dessa iniciativa, há também a necessidade de vários outros incentivos de melhoria da mobilidade urbana, por exemplo, maior incentivo à expansão e melhoria de meios de transporte coletivo, como o transporte sobre trilhos (trem e metrô) e ônibus. Adiciona-se a importância do transporte ativo, contribuindo para a diminuição da emissão de poluentes e também promovendo a atividade física na população (57).

Como limitação desse estudo, considera-se a limitação de se pesquisar dados públicos secundários, uma vez que, para os dados em saúde, temos a subnotificação e a falta de integração dos prontuários eletrônicos dos pacientes em um único Sistema de Informação. Para os dados meteorológicos e de poluentes, temos como fator limitante principal a falta 
de dados em alguns períodos e a utilização da média entre estações de monitoramento como valor representativo da concentração de poluentes e de parâmetros meteorológicos do município.

\section{Conclusão}

Nesse artigo, indicamos a diminuição dos níveis dos poluentes atmosféricos $\mathrm{MP}_{10}, \mathrm{SO}_{2}$ e $\mathrm{NO}_{2}$, no período estudado, com exceção do $\mathrm{O}_{3}$, poluente de origem secundária. Apesar do aumento da frota de automóveis do município de São Paulo, consideramos que a melhoria dos motores e combustíveis está relacionada ao decréscimo da concentração dos poluentes, mostrando a importância da manutenção de políticas públicas de longo prazo, na temática do controle das emissões de poluentes, como ocorre com o PROCONVE e o PROMOT.

Muitos estudos foram realizados nas últimas décadas fornecendo subsídios para a melhor tomada de decisão dos gestores, visando a redução dos efeitos nocivos dos poluentes atmosféricos na saúde. No entanto, as medidas e políticas que regulamentam e limitam a emissão desses poluentes ainda são obsoletas em comparação aos padrões instituídos em países desenvolvidos.

Adicionalmente, a partir dos resultados obtidos nesse estudo, contribuiu-se na atualização sobre os efeitos deletérios dos poluentes atmosféricos na saúde cardiorrespiratória. $\mathrm{O} \mathrm{NO}_{2}$ e o $\mathrm{MP}_{10}$ foram os poluentes que se mostraram mais significantes em todos os desfechos em saúde estudados nessa pesquisa, reforçando a necessidade de políticas e ações que visem o controle e a diminuição desses poluentes atmosféricos em grandes centros urbanos.

\section{Agradecimentos}

O presente trabalho foi realizado com o apoio da Fundação de Amparo à Pesquisa do Estado de São Paulo - FAPESP - Processo número 2019/14686-8.

\section{Conflitos de interesse}

Os autores declaram que não possuem conflitos de interesse.

\section{Referências}

1. Bai L, Wang J, Ma X, Lu H. Air Pollution Forecasts: An Overview. Int J Environ Res Public Health. 2018; 15(4): 780.

2. WHO. Ambient Air Pollution: a global assessment of exposure and burden of disease. Genebra: WORLD HEALTH ORGANIZATION, 131 p, 2016.

3. CETESB. Qualidade do ar no estado de São Paulo, 2020. Recurso eletrônico. São Paulo, 228p. Série Relatos. 2021. Disponível em: < https://cetesb.sp.gov.br/ar/wpcontent/uploads/sites/28/2021/05/Relatorio-de-Qualidade-do-Ar-no-Estado-de-Sao-Paulo-2020.pdf > Acesso em 10/10/2021.

4. Hoek G, Krishnan RM, Beelen R, Peters A, Ostro B, Brunekreef B, Kaufman JD. Long-term air pollution exposure and cardio- respiratory mortality: a review. Environ Health. 2013; 12(1): 43.

5. Chang CC, Chen PS, Yang CY. Short-term effects of fine particulate air pollution on hospital admissions for cardiovascular diseases: a case-crossover study in a tropical city. J Toxicol Environ Health A. 2015; 78(4): 267-77.

6. Xiong Q, Zhao W, Gong Z, Zhao W, Tang T. Fine Particulate Matter Pollution and Hospital Admissions for Respiratory Diseases in Beijing, China. Int J Environ Res Public Health. 2015; 12(9): 11880-92

7. Bravo MA, Son J, de Freitas CU, Gouveia N, Bell ML. Air pollution and mortality in São Paulo, Brazil: 
Effects of multiple pollutants and analysis of susceptible populations. J Expo Sci Environ Epidemiol. 2016; 26(2): 150-61.

8. Freitas CU, Ponce de Leon A, Juger W, Gouveia N. Poluição do ar e impactos na saúde em Vitória, Espírito Santo. Rev Saude Publica. 2016; 50:4.

9. To T, Zhu J, Larsen K, Simatovic J, Feldman L, Ryckman K, Gershon A, Lougheed MD, Licskai C, Chen H, Villeneuve PJ, Crighton E, Su Y, Sadatsafavi M, Williams D, Carlsten C; Canadian Respiratory Research Network. Progression from Asthma to Chronic Obstructive Pulmonary Disease. Is Air Pollution a Risk Factor? Am J Respir Crit Care Med. 2016; 194(4): 429-38.

10. Zúñiga J, Tarajia M, Herrera V, Urriola W, Gómez B, Motta J. Assessment of the Possible Association of Air Pollutants PM10, O3, NO2 With an Increase in Cardiovascular, Respiratory, and Diabetes Mortality in Panama City: A 2003 to 2013 Data Analysis. Medicine (Baltimore). 2016; 95(2): e2464.

11. Calderón-Garcidueñas L, Kulesza RJ, Doty RL, D'Angiulli A, Torres-Jardón R. Megacities air pollution problems: Mexico City Metropolitan Area critical issues on the central nervous system pediatric impact. Environ Res. 2015; 137:157-69.

12. Jung CR, Lin YT, Hwang BF. Ozone, particulate matter, and newly diagnosed Alzheimer's disease: a population-based cohort study in Taiwan. J Alzheimers Dis. 2015; 44(2): 573-84.

13. Angelici L, Piola M, Cavalleri T, Randi G, Cortini F, Bergamaschi R, Baccarelli AA, Bertazzi PA, Pesatori AC, Bollati V. Effects of particulate matter exposure on multiple sclerosis hospital admission in Lombardy region, Italy. Environ Res. 2016; 145:68-73.

14. Oudin A, Forsberg B, Adolfsson AN, Lind N, Modig L, Nordin M, Nordin S, Adolfsson R, Nilsson LG. Traffic-Related Air Pollution and Dementia Incidence in Northern Sweden: A Longitudinal Study. Environ Health Perspect. 2016; 124(3): 306-12.

15. Choi H, Rauh V, Garfinkel R, Tu Y, Perera FP. Prenatal exposure to airborne polycyclic aromatic hydrocarbons and risk of intrauterine growth restriction. Environ Health Perspect. 2008; 116(5): 65865.

16. Parker JD, Mendola P, Woodruff TJ. Preterm birth after the Utah Valley Steel Mill closure: a natural experiment. Epidemiology. 2008; 19(6): 820-3.

17. Darrow LA, Klein M, Flanders WD, Waller LA, Correa A, Marcus M, Mulholland JA, Russell AG, Tolbert PE. Ambient air pollution and preterm birth: a time-series analysis. Epidemiology. 2009; 20(5): 689-98.

18. Rich DQ, Demissie K, Lu SE, Kamat L, Wartenberg D, Rhoads GG. Ambient air pollutant concentrations during pregnancy and the risk of fetal growth restriction. J Epidemiol Community Health. 2009; 63(6): 488-96.

19. IBGE - Instituo Brasileiro de Geografia e Estatística. Cidades e Estados, 2021. São Paulo. Disponível em: < https://www.ibge.gov.br/cidades-e-estados/sp/sao-paulo.html > Acesso em 10/10/2021.

20. SEADE. Imprensa - Seade disponibiliza projeções de população e domicílios até 2050. Fundação Sistema Educacional de Análise de Dados - Portal de Estatísticas do Estado de São Paulo, 2017. Disponível em: <https://www.seade.gov.br/seade-disponibiliza-projecoes-de-populacao-e-domiciliosate-2050/>. Acesso em: 04/07/2020.

21. DENATRAN - Ministério da Infraestrutura, Departamento Nacional de Trânsito - 2018. Disponível em <https://cidades.ibge.gov.br/brasil/sp/saopaulo/pesquisa/22/28120?tipo=grafico\&indicador=28122>. Acesso em 29/09/2020.

22. Prefeitura de São Paulo. Demografia - Tabelas. 2018. Disponível em: <https://www.prefeitura.sp.gov.br/cidade/secretarias/urbanismo/dados_estatisticos/info_cidade/demo grafia/index.php?p=260265>. Acesso em: 29/09/2020.

23. Marôco JA. Estatística com o SPSS Statistics: $7^{a}$ edição. Report Number Lda, 2018.

24. Rodopoulou S, Samoli E, Chalbot MG, Kavouras IG. Air pollution and cardiovascular and respiratory emergency visits in Central Arkansas: A time-series analysis. Sci Total Environ. 2015; 536: 872-879

25. Abe KC, Santos GMS, Coêlho MSZS, Miraglia, SGEK. PM10 Exposure and Cardiorespiratory Mortality - Estimating the Effects and Economic Losses in São Paulo, Brazil. Aerosol Air Qual. Res. 2018; 18: 3127-3133.

26. Chen Y, Zang L, Du W, Xu D, Shen G, Zhang Q, Zou Q, Chen J, Zhao M, Yao D. Ambient air pollution of particles and gas pollutants, and the predicted health risks from long-term exposure to PM2.5 in Zhejiang province, China. Environ Sci Pollut Res Int. 2018; 25(24): 23833-23844

27. SÃO PAULO. Decreto $n^{\circ} 59.113$, de 23 de abril de 2013. Estabelece novos padrões de qualidade do ar e dá providências correlatas. São Paulo: Assembleia Legislativa do Estado de São Paulo, 2013. Disponível em: <https://www.al.sp.gov.br/repositorio/legislacao/decreto/2013/decreto-5911323.04.2013.html>. Acesso em: 10/10/2021.

28. BRASIL, MMA. PROCONVE - Programa de controle de poluição do ar por veículos automotores. Ministério do Meio Ambiente, 2013. Disponível em: 
<https://www.mma.gov.br/estruturas/163/_arquivos/proconve_163.pdf>. Acesso em: 29/09/2020.

29. CETESB. Companhia Ambiental do Estado de São Paulo. Diretoria de Engenharia e Qualidade Ambiental. Informação Técnica $\mathrm{n}^{\circ}$ 11/18, 2018. Disponível em <https://cetesb.sp.gov.br/wpcontent/uploads/2018/12/17.pdf>. Acesso em 29/09/2020.

30. BRASIL, MMA. PROMOT - Programa de controle da poluição do ar por motociclos e veículos similares. Ministério do Meio Ambiente, 2015. Disponível em: <https://www.mma.gov.br/estruturas/163/_arquivos/promot_163.pdf>. Acesso em: 29/09/2020.

31. CETESB. Companhia Ambiental do Estado de São Paulo. Diretoria de Engenharia e Qualidade Ambiental. Informação Técnica $\mathrm{n}^{\circ}$ 02/19/ETH/ET, 2019. Disponível em: < https:/cetesb.sp.gov.br/wp-content/uploads/2019/05/An\%C3\%A1lise-proposta-M5-aprovada-noCONAMA.pdf $>$. Acesso em: 29/09/2020.

32. COHEN, Jacob. Statistical power analysis for the behavioral sciences - Second Edition. Lawrence Erlbaum Associates, $1988 . \quad$ Disponível em: <http://www.utstat.toronto.edu/ brunner/oldclass/378f16/readings/CohenPower.pdf $>$.

33. Costa AF, Hoek G, Brunekreef B, Ponce de Leon AC. Air Pollution and Deaths among Elderly Residents of São Paulo, Brazil: An Analysis of Mortality Displacement. Environ Health Perspect. 2017; 125(3):349-354

34. Ribeiro AG, Baquero OS, Almeida SL, Freitas CU, Cardoso MRA, Nardocci AC. Influence of vehicular traffic density on hospital admissions due to respiratory tract cancer in the city of São Paulo, Brazil. Cadernos de Saúde pública. 2019; 35 (1): e00128518.

35. Pinheiro Sde L, Saldiva PH, Schwartz J, Zanobetti A. Isolated and synergistic effects of PM10 and average temperature on cardiovascular and respiratory mortality. Rev Saude Publica. 2014; 48(6): 8818.

36. Nascimento AP, Santos JM, Mill JG, Souza JB, Reis NC, Reisen VA. Associação entre concentração de partículas finas na atmosfera e doenças respiratórias agudas em crianças. Revista de Saúde Pública. 2017; 51: 3 .

37. Ribeiro AG, Baquero OS, Freitas CU, Chiaravalotti Neto F, Cardoso MRA, Latorre MDRDO, Nardocci AC. Incidence and mortality risk for respiratory tract cancer in the city of São Paulo, Brazil: Bayesian analysis of the association with traffic density. Cancer Epidemiol. 2018; 56: 53-59.

38. Ho HC, Wong MS, Yang L, Chan TC, Bilal M. Influences of socioeconomic vulnerability and intraurban air pollution exposure on short-term mortality during extreme dust events. Environ Pollut. 2018; 235:155-162

39. Zheng PW, Wang JB, Zhang ZY, Shen P, Chai PF, Li D, Jin MJ, Tang ML, Lu HC, Lin HB, Chen K. Air pollution and hospital visits for acute upper and lower respiratory infections among children in Ningbo, China: A time-series analysis. Environ Sci Pollut Res Int. 2017; 24(23): 18860-18869..

40. Klompmaker JO, Janssen N, Andersen ZJ, Atkinson R, Bauwelinck M, Chen J, de Hoogh K, Houthuijs D, Katsouyanni K, Marra M, Oftedal B, Rodopoulou S, Samoli E, Stafoggia M, Strak M, Swart W, Wesseling J, Vienneau D, Brunekreef B, Hoek G. Comparison of associations between mortality and air pollution exposure estimated with a hybrid, a land-use regression and a dispersion model. Environ Int. 2021; 146: 106306.

41. Huang S, Li H, Wang M, Qian Y, Steenland K, Caudle WM, Liu Y, Sarnat J, Papatheodorou S, Shi L. Long-term exposure to nitrogen dioxide and mortality: A systematic review and meta-analysis. Sci Total Environ. 2021; 776:145968.

42. Huangfu P, Atkinson R. Long-term exposure to NO2 and $\mathrm{O} 3$ and all-cause and respiratory mortality: A systematic review and meta-analysis. Environ Int. 2020; 144: 105998.

43. Nuvolone D, Petri D, Voller F. The effects of ozone on human health. Environ Sci Pollut Res Int. 2018; 25(9): 8074-8088.

44. Wu Y, Li R, Cui L, Meng Y, Cheng H, Fu H. The high-resolution estimation of sulfur dioxide (SO2) concentration, health effect and monetary costs in Beijing. Chemosphere. 2020; 241:125031.

45. ANFAVEA. Carta Digital - Dez/2020 - Ed. 415. Associação Nacional dos Fabricantes de Veículos Automotores. 2020

46. Abe KC, Miraglia SG. Health Impact Assessment of Air Pollution in São Paulo, Brazil. Int J Environ Res Public Health. 2016; 13(7): 694.

47. Chanel O, Perez L, Künzli N, Medina S; Aphekom group. The hidden economic burden of air pollution-related morbidity: evidence from the Aphekom project. Eur J Health Econ. 2016; 17(9): 1101-1115.

48. Mueller N, Rojas-Rueda D, Basagaña X, Cirach M, Cole-Hunter T, Dadvand P, Donaire-Gonzalez D, Foraster M, Gascon M, Martinez D, Tonne C, Triguero-Mas M, Valentín A, Nieuwenhuijsen M. Health impacts related to urban and transport planning: A burden of disease assessment. Environ Int. 2017; 107: 243-257. 
R. M. P. de Godoy et al. / Vittalle v. 33 n. 3 (2021) 33-46

49. Pereira JAG. Carga fiscal inviabiliza venda de carro elétrico. Página 22. 2013; 75: 13.

50. Júnior SRR, Coeli, MA. Isenção fiscal para veículos elétricos e híbridos [Trabalho de Conclusão de Curso]. Repositório da Universidade de Uberaba (Uniube); Coleção 2018/2. 14 de novembro de 2018. Disponível

em: <https://repositorio.uniube.br/bitstream/123456789/934/1/ISEN\%c3\%87\%c3\%830\%20FISCAL\%20 PARA\%20VE\%c3\%8dCULOS\%20EL\%c3\%89TRICOS\%20E\%20H\%c3\%8dBRIDOS.pdf>. Acessado em 16/12/2021.

51. Zaneti L. Diagnóstico dos produtos e serviços complementares para a adoção dos carros elétricos e híbridos no Brasil [Trabalho de Conclusão de Curso]. Florianópolis: Departamento de Engenharia de Produção e Sistemas, Universidade Federal de Santa Catarina, 2018.

52. Moreira JR, Coelho ST, Velazquez SMSG, Santos MA, Melo EH, Elmadjian PHB. Veículos elétricos híbridos e a emissão de poluentes. XVII Simpósio Internacional de Engenharia Automotiva, São Paulo, SP, 2009.

53. Loch E, Henkes JA. Alternativas para reduzir a poluição gerada por automóveis a partir do uso de energias renováveis. Revista Gestão \& Sustentabilidade Ambiental. 2014; 3(1): 565-583.

54. Parente CG, Souza DAC, Sampaio CB, Barbosa SG, Oliveira DS. Estratégias para mitigar a emissão de poluentes no setor de carros particulares. In: VII Congresso Brasileiro de Energia Solar-CBENS 2018. 2020.

55. Vilaca NMCAA, Figueiredo VN, Oliveira LB, Ferreira VH, Fortes MZ, Correia WF, Pacheco OLC. Smart city-caso de implantação em Búzios-RJ. Revista Sodebras. 2014; 9(98): 16-22.

56. Xavier PCR. Eletroposto em Uberlândia: preparando a cidade para automóveis não poluentes [Trabalho de conclusão de Curso]. Uberlândia: Graduação em Arquitetura e Urbanismo, Universidade Federal de Uberlândia, Uberlândia, 2020. 77p.

57. Rojas-Rueda D, de Nazelle A, Andersen ZJ, Braun-Fahrländer C, Bruha J, Bruhova-Foltynova H, Desqueyroux H, Praznoczy C, Ragettli MS, Tainio M, Nieuwenhuijsen MJ. Health Impacts of Active Transportation in Europe. PLoS One. 2016; 11(3): e0149990. 